\title{
Probing the Dark Ages with the Square Kilometer Array
}

\author{
C.L. Carillia ${ }^{\mathrm{a}}$, S. Furlanetto ${ }^{\mathrm{b}}$, F. Briggs ${ }^{\mathrm{c}}$, M. Jarvis, S. Rawlings ${ }^{\mathrm{d}}$, H. Falcke ${ }^{\text {e }}$

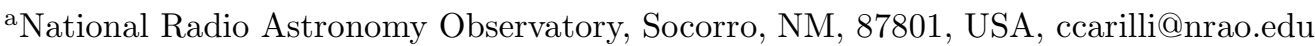 \\ ${ }^{\mathrm{b}}$ Caltech, Pasadena, CA, USA \\ ${ }^{\mathrm{c}}$ Australia Telescope National Facility, Sydney, Australia \\ dAstrophysics, Dept. of Physics, Keble Road, Oxford OX1 3RH, UK \\ eASTRON, P.O. Box 2, 7990 AA Dwingeloo, The Netherlands
}

The epoch of reionization (EoR) sets a fundamental benchmark in cosmic structure formation, corresponding to the formation of the first luminous objects that act to ionize the neutral intergalactic medium (IGM). Recent observations at near-IR and radio wavelengths imply that we are finally probing into this key epoch of galaxy formation at $z \geq 6$. The Square Kilometer Array (SKA) will provide critical insight into the EoR in a number of ways. First, the ability of the SKA to image the neutral IGM in $21 \mathrm{~cm}$ emission is a truly unique probe of the process of reionization, and is recognized as the next necessary and fundamental step in our study of the evolution of large scale structure and cosmic reionization. Second, study of HI $21 \mathrm{~cm}$ absorption toward the first radio loud objects probes small to intermediate scale structure in the neutral 'cosmic web', as well as HI in the first collapsed structures (proto-disks and mini-halos). And third, the incomparable sensitivity of the SKA allows for the study of the molecular gas, dust, and star formation activity in the first galaxies, as well as the radio continuum emission from the first accreting massive black holes. Such objects will be obscured at optical wavelengths due to absorption by the neutral IGM.

\section{Introduction}

After recombining at $z \sim 1000$, the intergalactic medium (IGM) remains neutral until the formation of the first luminous sources in the universe. These act to reionize the universe, leading to the fully ionized IGM seen today (neutral fraction $\sim 10^{-5}$ ). The epoch of cosmic reionization (EoR) therefore sets a fundamental benchmark in cosmic structure formation, corresponding to the formation of the first luminous objects (star forming galaxies and/or accreting massive black holes).

The last few years has seen a revolution in our understanding of the EoR, in particular, the first observational constraints on the redshift for the EoR. The discovery of the Gunn-Peterson absorption trough in the spectra of the most distant quasars $(z>6)$, corresponding to Ly $\alpha$ absorption by the neutral IGM, implies that we have finally probed into the EoR [43]. These data show that the neutral fraction of the IGM decreases rapidly over a short cosmic time, from $>10^{-3}$ at $z=6.3$, to $<10^{-4}$ at $z=5.8$. A rapid transition is supported by a number of lines of evidence, including: (i) studies of the thermal state of the IGM at high redshift via high $z$ Ly $\alpha$ forest observations [22], (ii) observations of cosmic Stromgren spheres and surfaces around the highest redshift QSOs 414628, and (iii) $\Lambda$ CDM galaxy formation models normalized to known high $z$ galaxy populations [18. However, recent CMB large scale polarization measurements by WMAP suggest a significantly ionized IGM at $z \sim 10$ to 20 [26. Taken together, current observational constraints suggest that cosmic reionization had a fairly complex history, extending from $z=6$ to 20 [7.

While the discovery of the GP effect, and the large scale polarization of the $\mathrm{CMB}$, are clearly monumental achievements in observational cosmology, both techniques have limitations. For the 
GP effect, the IGM becomes opaque for neutral fractions $\geq 10^{-3}$, such that the technique loses it's diagnostic power once the IGM becomes a small fraction neutral. For the CMB, the large scale polarized signal is an integral measure of the universal optical depth to Thompson scattering, and can be fit with myriad reionization scenarios [14. The SKA will play a pivotal role in determining the epoch, and physics, of cosmic reionization in two ways: (1) through direct observations of the neutral IGM via the HI $21 \mathrm{~cm}$ line, and (2) through continuum and line observations of the first luminous objects - stars, galaxies and black holes.

The ability of the SKA to image the neutral IGM in $21 \mathrm{~cm}$ emission is a truly unique probe of the process of reionization, and is recognized as the next necessary and fundamental step in our study of cosmic reionization. Likewise, study of HI $21 \mathrm{~cm}$ absorption toward the first radio loud objects probes small to intermediate scale structure in the neutral 'cosmic web', as well as HI in the first collapsed structures (proto-disks and mini-halos). Observations of the HI $21 \mathrm{~cm}$ line are a direct probe of the evolution of the neutral IGM, and provide key diagnostics on the ionization and thermal state of the IGM, the formation of large and small scale structure, and the nature of the first luminous objects (AGN or stars).

The incomparable sensitivity of the SKA allows for the study of the molecular gas, dust, and star formation activity in the first galaxies, as well as the radio continuum emission from the first accreting massive black holes. It is important to keep in mind that, due to the on-set of GP absorption at $z \sim 6$, observations of objects within the EoR are limited to wavelengths longer than 0.9 micron. Hence study of the first galaxies and AGN is the exclusive realm of near-IR to radio astronomy (and X-rays).

The study of the evolution of the neutral IGM, and the first luminous objects, has been selected as one of the key science programs for the SKA. Details of these studies can be found in contributions by Furlanetto, Carilli, Blain, Falcke, and Jarvis in this volume. We review this material herein.

\section{Observing the $\mathrm{HI} 21 \mathrm{~cm}$ line from the Epoch of Reionization}

\subsection{Tomography}

The study of the redshifted HI $21 \mathrm{~cm}$ line at meter wavelengths with the SKA provides a direct probe of the pristine neutral IGM within the EoR. Unlike studies of the Ly $\alpha$ forest, $21 \mathrm{~cm}$ tomography does not suffer from saturation when the neutral fraction is large, so it is ideal for studying the middle and early stages of reionization. Moreover, because it is a spectral line observation, it provides a complete time history of reionization rather than an integral constraint, such as the optical depth to electron scattering of the CMB. The major difficulty with the technique the weak signals - makes this an ideal project for the SKA.

The first consideration of detecting the $21 \mathrm{~cm}$ signal from the EoR entailed searching for a global step in the background temperature due to the transition from a neutral to ionized IGM [37. Given this is a full-sky measurement, the sensitivity is independent of the telescope area. The most recent treatment of this problem can be found in [15. This is a difficult observation, requiring detecting a change in the overall background spectral index at the $0.1 \%$ level, and the difficulty is excerbated if the EoR extends over a large redshift range.

A more interesting application is to map out variations in the neutral hydrogen density, either across the sky or in frequency space. Such "tomography" studies allows one to reconstruct the time evolution of reionization in an entirely unique manner. The idea is to measure the fluctuating brightness temperature of the IGM gas, which depends in turn on the local overdensity, neutral fraction, and spin temperature. In general we expect three distinct observational epochs. Before the first luminous sources form, we should see weak fluctuations from the "protocosmic web" and virialized gas in dark matter "minihalos" 2423. After these sources turn on, Ly $\alpha$ photons flood the universe and render the entire IGM visible against the CMB. Depending on the thermal history of the gas, this era can be seen in either absorption or emission, so this era 
can be used to study the first luminous sources in the universe. Once the IGM is heated to well above the CMB temperature (either through radiation or shock heating), temperature fluctuations no longer affect the brightness temperature and we see only fluctuations in the density and ionized fraction.

We expect most of the interesting phases of reionization to take place during this last era. Strömgren spheres surrounding ionizing sources rapidly dominate the fluctuations (see Fig. 1). However, our current understanding of this process is poor and measurements of reionization are crucial for advancing our understanding of this landmark event. In particular, we would like to know how the HII regions grow around sources, and which sources are most responsible for reionization. The contribution by Furlanetto (this volume) describes several theoretical studies of the signals expected during reionization. In all models, we expect fluctuations of order $1-10 \mathrm{mK}$ on scales of one to several arcminutes, with the characteristic scale increasing as reionization proceeds 13. The growth of these regions, as well as the mean signal, directly measures the entire ionization history, the morphology of the HII regions, and the path by which the IGM is ionized (for example, whether voids or dense regions are ionized first). The expected signal is thus rich in potential physical diagnostics of both the IGM and the first generations of luminous sources.

The SKA will be the first instrument with adequate sensitivity to image fluctuations over the entire set of angular scales relevant to these observations, especially near the beginning of reionization when the HII regions are small (near or somewhat below an arcminute in size). While instruments such as $\mathrm{PAST}^{1}$ and $\mathrm{LOFAR}^{2}$ should be able to measure some of the statistical properties of neutral gas and possibly map the sky on coarse angular scales, the SKA offers a unique opportunity to image the evolution of large-scale structures during the EoR (Figure 1). The expected frequency range of $100 \mathrm{MHz}$ to $200 \mathrm{MHz}$ $(z=6$ to 13$)$ is sufficient to place powerful con-

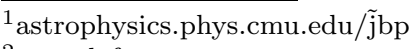

${ }^{2}$ www.lofar.org straints on the ionization history, though measurements of "first light" may require lower frequencies. One of the major challenges in recovering this signal is foreground contamination from Galactic synchrotron radiation and extragalactic point sources. However, there are several promising techniques to eliminate this contamination. Zaldariagga et al. 47 showed that the smooth spectra of these foregrounds allow efficient cleaning through differencing maps closely spaced in frequency. Morales \& Hewitt 29 showed that symmetries in the spectral domain also allow foreground removal. While technically challenging, we expect that $21 \mathrm{~cm}$ tomography will revolutionize our understanding of the high-redshift universe.

\subsection{Absorption}

Observing HI $21 \mathrm{~cm}$ emission from the EoR implies studying large scale structure (cluster scales and larger). A number of groups have recently considered the possibility of studying smaller scale structure in the neutral IGM by looking for HI $21 \mathrm{~cm}$ absorption toward the first radio-loud objects (AGN, star forming galaxies, GRBs).

Carilli et al. 6 use numerical simulations to predict the HI $21 \mathrm{~cm}$ absorption profile of the 'cosmic web' prior to reionization. For example, for a source at $z=10$, they predict an average optical depth due to $21 \mathrm{~cm}$ absorption of about $1 \%$, corresponding to the 'radio Gunn-Peterson effect'. They also find about five narrow (few $\mathrm{km} / \mathrm{s}$ ) absorption lines per $\mathrm{MHz}$ with optical depths of a few to $10 \%$ (Figure 2). These latter lines are equivalent to the Ly $\alpha$ forest seen after reionization, and correspond to over-densities evolving in the linear regime $(\delta \leq 10)$. While significant questions remain about simulating the thermal state of the IGM to such detail, the simple point remains that, while the $\operatorname{Ly} \alpha$ lines from such structures are highly saturated, the (much) lower $21 \mathrm{~cm}$ oscillator strength makes the IGM translucent prior to reionization.

It is possible that, due to the lower cosmic Jean's mass prior to reionization, the majority of the HI is contained in mini-halos, ie. collapsed structures with $\delta>100$ and masses $\leq 10^{7} \mathrm{M}_{\odot}$. Furlanetto \& Loeb 12 have considered this pos- 


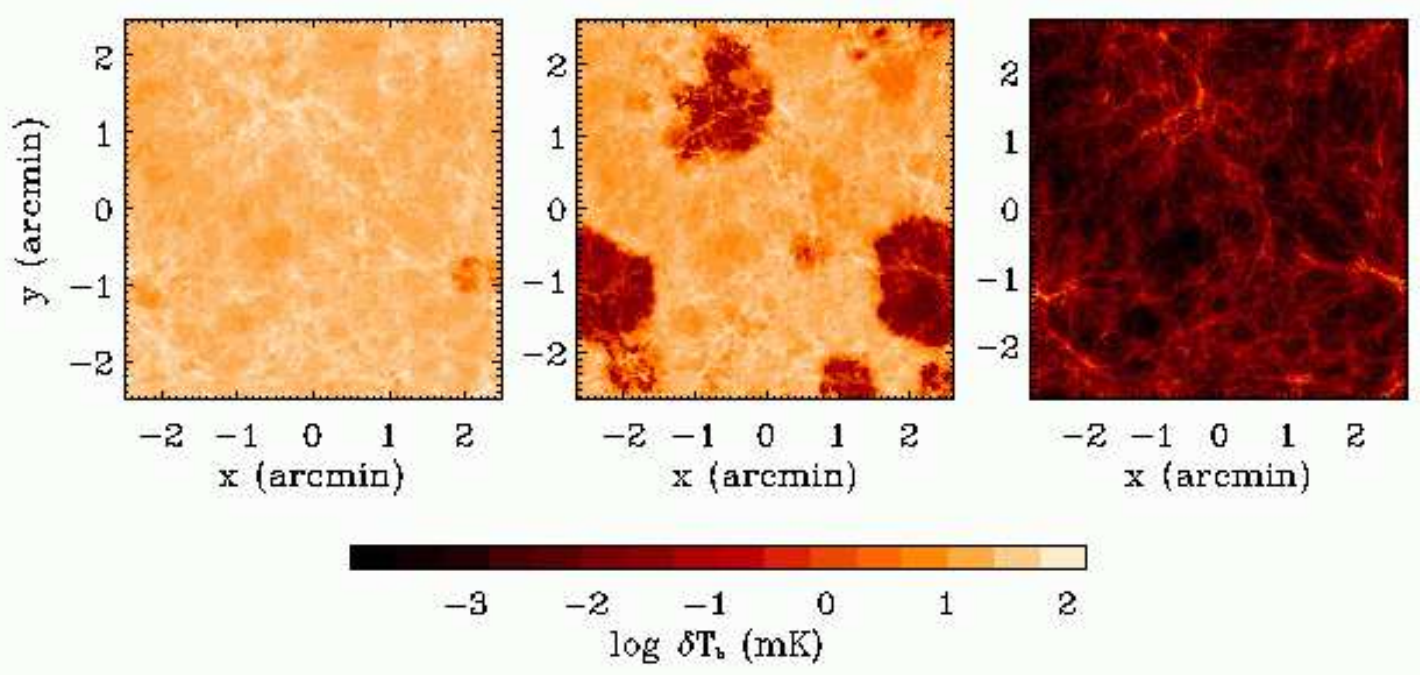

Figure 1. The brightness temperature of the $21 \mathrm{~cm}$ transition at several redshifts, as predicted by the "late reionization" simulation analyzed in [13] (see contribution by Furlanetto, this volume). Each panel corresponds to the same slice of the simulation box (with width $10 h^{-1}$ comoving Mpc and depth $\Delta \nu=0.1$ $\mathrm{MHz}$ ), at $z=12.1,9.2$, and 7.6, from left to right. The three epochs shown correspond to the early, middle, and late stages of reionization in this simulation.

sibility and predict a similar HI $21 \mathrm{~cm}$ absorption line density due to gas in mini-halos as that expected for the $21 \mathrm{~cm}$ forest.

The predicted absorption signal depends on the excitation state of the HI. This in turn depends on IGM pre-heating, including possible early reionization (and subsequent recombination), resonant scattering of Ly $\alpha$ photons, and heating by soft Xrays. Cen [7] shows that the presence or absence of HI absorption by mini-halos, or the cosmic web, would be a telling diagnostic of early IGM heating mechanisms.

Furlanetto \& Loeb [12] also consider the expected $21 \mathrm{~cm}$ absorption profiles for proto-disk galaxies. While such absorption lines will be rare $\left(10^{4}\right.$ times less frequent than the $21 \mathrm{~cm}$ forest lines), the optical depths may be large enough that the lines could be observed toward faint radio sources, in particular, gamma ray burst radio after-glows within the host galaxy.

An important caveat in these calculations is the assumption of radio loud sources during the EoR. This question has been considered in detail by
Carilli et al. 6] and Haiman et al. 17. They show that current models of radio-loud AGN evolution predict between 0.05 and 1 radio sources per square degree at $z>6$ with $\mathrm{S}_{150 \mathrm{MHz}} \geq 6$ mJy, adequate for EoR HI $21 \mathrm{~cm}$ absorption studies with the SKA (see also section 4).

\section{Study of the first luminous objects}

Gunn-Peterson absorption during the EoR $(z \geq 6)$ precludes observations of objects at wavelengths longer than 0.9 micron. Hence study of the first galaxies and AGN is the exclusive realm of near-IR to radio astronomy. The last few years has seen a revolution in the number of objects discovered at $z>6$ using near-IR and radio imaging and spectroscopy, including star forming galaxies 39 21 25. and AGN [11].

The recent discovery of molecular line emission, thermal emission from warm dust, and radio syncrotron emission, from the most distant QSO $(z=6.4 ; 2414)$, implies very early enrichment of heavy elements and dust in galaxies, presumably via star formation, within $0.8 \mathrm{Gyr}$ of 


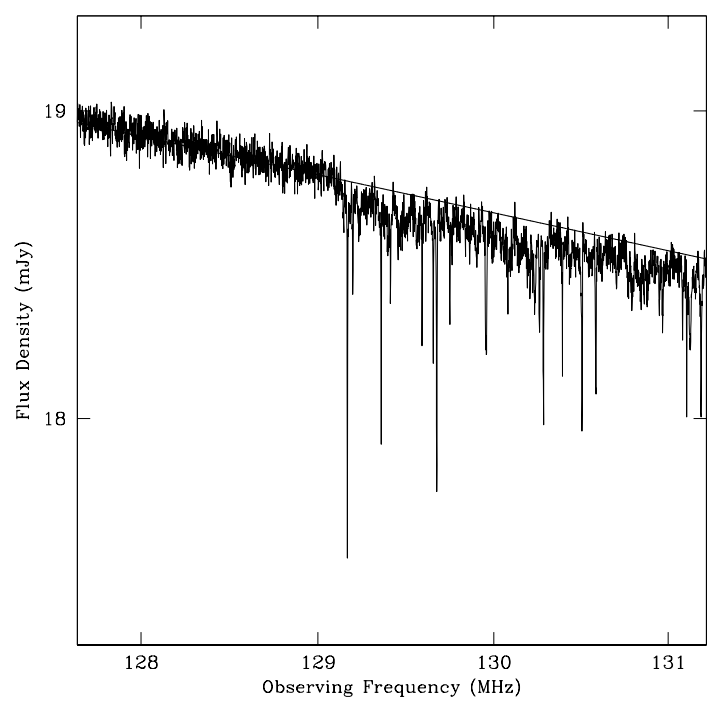

Figure 2. The simulated spectrum of a source with $\mathrm{S}_{120}=20 \mathrm{mJy}$ at $z=10$ using a spectral model based on the powerful radio galaxy Cygnus A and assuming HI $21 \mathrm{~cm}$ absorption by the IGM (see contribution by Carilli, this volume, [6]). Thermal noise has been added using the specifications of the SKA and assuming 10 days integration with $1 \mathrm{kHz}$ wide spectral channels. The onset of absorption by the neutral IGM is seen at $129 \mathrm{MHz}$, corresponding to the $\mathrm{HI} 21 \mathrm{~cm}$ line at $z=10$.

the big bang (Figure 3). Unfortunately, such experiments stretch current instrumentation to the extreme limit, such that only rare and pathologic objects are detectable, ie. hyperluminous IR galaxies with $L_{F I R}>10^{13} \mathrm{~L}_{\odot}$. The two orders of magnitude increase in sensitivity afforded by the SKA will enable study of the first 'normal' galaxies within the EoR in a number of ways.

The SKA operating at short $\mathrm{cm}$ wavelengths $(\sim 20 \mathrm{GHz})$ allows for detailed molecular line imaging of galaxies in the high- $z$ universe. The high redshifts involved mean that the standard $\mathrm{mm}$ molecular transitions will be available for study with the SKA, eg. observations at $20 \mathrm{GHz}$ correspond to $\mathrm{CO} 1-0$ at $z=5, \mathrm{CO} 2-1$ at $z=10$, and HCN $1-0$ at $z=3.5$. Such studies reveal the physics and chemistry of molecular gas reservoirs required for star formation, and provide a unique probe of gas dynamics and dynamical masses of the first galaxies [?]. Also, radio continuum studies with nanoJy sensitivity in the frequency range 1 to $10 \mathrm{GHz}$ will present a dust-unbiased view of star formation in these systems.

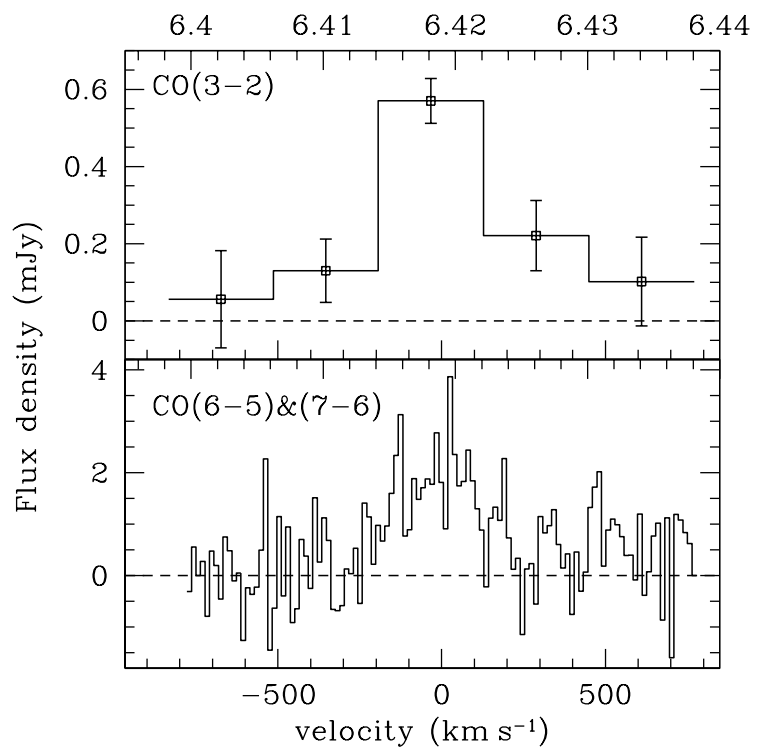

Figure 3. The CO line emission from the most distant QSO, $1148+5251$ at $z=6.42$ [411]. The 3-2 line was observed with the Very Large Array at $47 \mathrm{GHz}$, while the higher order transitions were observed with the Plateau de Bure interferometer. The implied molecular gas mass is $2.2 \times 10^{10}$ $\mathrm{M}_{\odot}$.

Note that ALMA also has the potential for studying molecular emission from high redshift objects, but only in systems where very highorder transitions are excited (eg. observations at $90 \mathrm{GHz}$ correspond to CO 7-6 at $z=8$ ), and with less sensitivity than SKA, even for thermally excited lines. In general, SKA and ALMA will pro- 
vide complementary information on the low and high order molecular emission from galaxies at intermediate redshifts ( $z=3$ to 6$)$, while at the highest redshifts, ie. into the $\operatorname{EoR}(z>6)$, the SKA becomes the premier instrument for studying the molecular content of early galaxies (see contribution by Blain, this volume). For example, Carilli \& Blain [3] show that in optimal surveys at $20 \mathrm{GHz}$ the SKA will detect of order 15 sources per hour in CO 1-0 emission at $z \sim 4$.

As a concrete example of the types of objects that might be studied, Pello et al. 34 have recently presented evidence in the near-IR for a possible strongly lensed $z \sim 10$ galaxy. Although the detection appears to be spurious [42, the proposed source properties may be considered characteristic of the first generation of galaxies that will be studied in deep near-IR surveys. The apparent UV luminosity is $4 \times 10^{11} \mathrm{~L}_{\odot}$. Making the standard dust correction for typical high redshift star forming galaxies (ie. Ly-break galaxies) then implies an apparent (FIR) luminosity of $2 \times 10^{12}$ $\mathrm{L}_{\odot}$. Assuming a line width of $300 \mathrm{~km} / \mathrm{s}$, the predicted CO 2-1 velocity integrated flux density is $4.5 \mathrm{mJy} \mathrm{km} \mathrm{s}^{-1}$ at $21 \mathrm{GHz}$. This $\mathrm{CO}$ emission line could be detected (at $5 \sigma$ ) with the SKA in 10 minutes. The (non-thermal) radio continuum emission is about $1 \mu \mathrm{Jy}$ at $1.4 \mathrm{GHz}$, which could be detected with the SKA in about 15 minutes. And to emphasize complementarity with future instrumentation, the predicted thermal emission from warm dust at $250 \mathrm{GHz}$ is $0.6 \mathrm{mJy}$, which can be detected with ALMA in a few minutes as well. If we remove the effect of strong lensing the required integration times increase by about two orders of magnitude, but that still means only about 20 hours integration to detect the first galaxies in the universe. Indeed, Hopkins [20] predicts that, in an $8 \mathrm{hr}$ integration with the SKA at $1.4 \mathrm{GHz}$, one would detect of order 7000 star forming galaxies at $z>5$ in the $1 \operatorname{deg}^{2}$ FoV of the SKA (see van der Hulst et al this volume, although cf. Jackson, this volume).

Figure 4 shows the spectrum of the active star forming galaxy Arp 220 at a number of redshifts, compared to the sensitivity of future instruments. The SKA is well matched to future instruments such as ALMA and the JWST in terms of study- ing the first luminous objects in the universe, allowing for a panchromatic view of the earliest stages of galaxy formation. Such a broad view will reveal the complete suite of physical processes in these systems, including stars, star formation, the interstellar medium (dust and gas), and the galaxy dynamics (dark matter), in objects forming within 0.5 Gyr of the big bang.

Lastly, an important aspect of the molecular line observations of galaxies within the EoR is that they give the most accurate redshifts (by far) for the host galaxies. Typical high ionization broad metal emission lines from QSOs are notoriously uncertain in terms of the host galaxy redshifts, with offsets typically on the order of $10^{3}$ $\mathrm{km} \mathrm{s}^{-1}$ [36], while Ly $\alpha$ emission lines are affected severely by absorption. Accurate host galaxy redshifts are crucial in the calculation of the size of cosmic Stromgren spheres around objects within the EoR, since these sizes are derived from the redshift difference between the host galaxy and the on-set of GP absorption [4]. The sizes of these ionized regions have been used to constrain the IGM neutral fraction [46, setting a lower limit to the neutral fraction of 0.1 at $z \sim 6.4$, two orders of magnitude more stringent than the lower limit set by the GP effect.

\section{The First AGN}

\subsection{Extended radio sources}

Within the EoR, hierarchical clustering will begin to yield the first dark-matter halos massive enough to host supermassive black holes (SMBH). During the major growth phases of these objects, they will shine as QSOs, although much of the radiation may be absorbed locally by dense neutral material in the dark-matter halo, or in the more diffuse neutral IGM. The mass-doubling timescale for an Eddington-rate-limited SMBH is $\sim 4 \times 10^{7} \mathrm{yr}$, which is not much smaller than the time interval between the epoch at which a suitably massive SMBH could first have formed and the end of the EoR (Figure 6; 45]). It is therefore reasonable to assume that most SMBHs within the EoR will be observed accreting at some high fraction of the Eddington-limited rate, although the possibility that an important propor- 


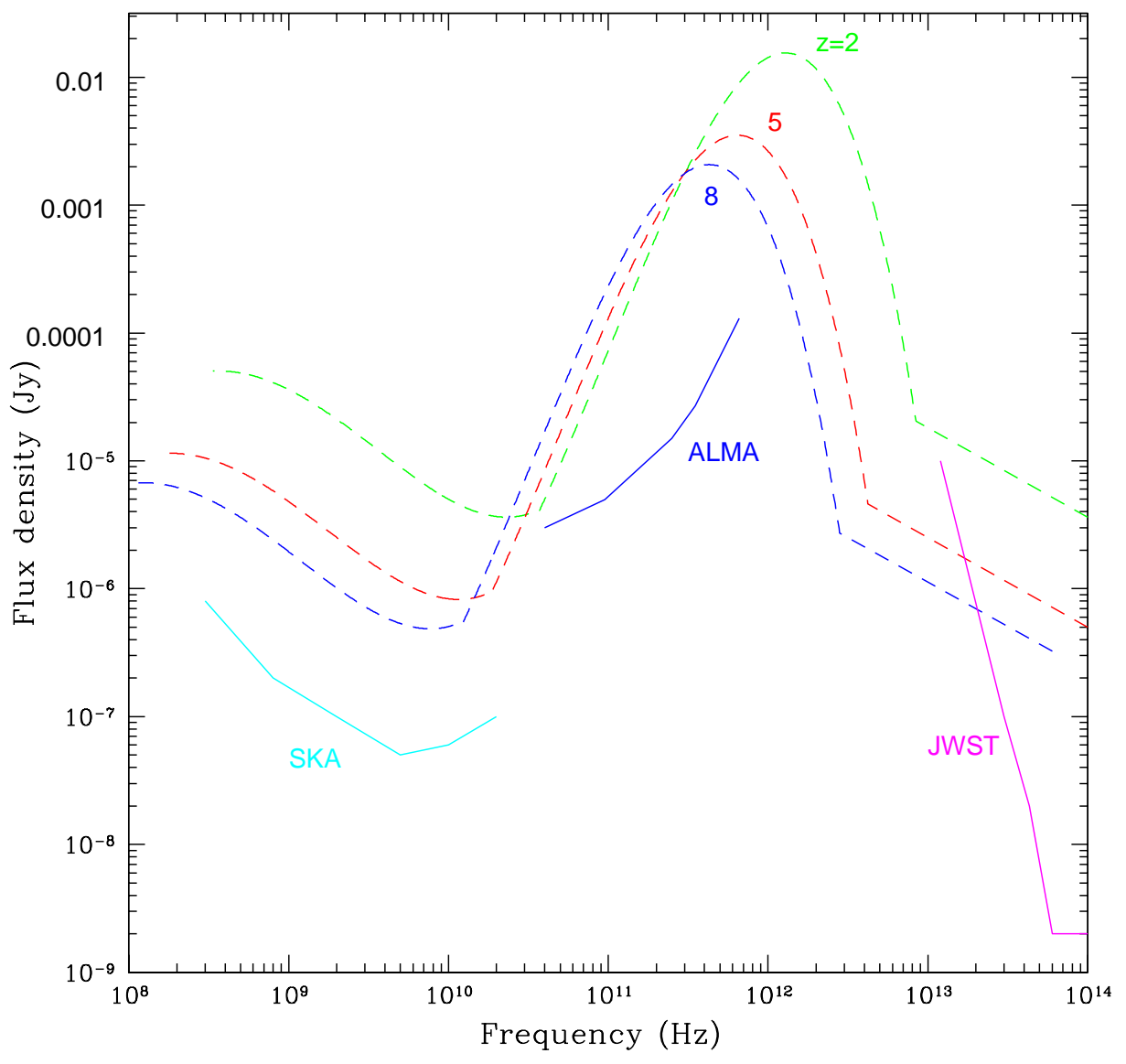

Figure 4. The dash lines show the spectrum of the active star forming galaxy Arp $220\left(L_{F I R}=1.3 \times 10^{12}\right.$ $\left.\mathrm{L}_{\odot}\right)$ at three redshifts $(z=2,5,8)$. The solid lines show the rms sensitivity of future instruments (in $1 \mathrm{hr})$ at $\mathrm{cm}$ through near-IR wavelengths. 
tion of black hole growth occurs in short-lived super-Eddington-accreting bursts 8 can never be easily discounted. Since the SKA can penetrate the huge optical depths common in the EoR, and since its will have unparalleled sensitivity to quasar activity over wide fields of view (FOV), it will become the premier instrument for understanding quasar (i.e. SMBH) feedback within the EoR.

Some small fraction $(\sim 0.01)$ of these accreting SMBH will generate powerful radio jets (radioloud quasars). Their radio luminosities are immense so a significant fraction of those observable in the EoR have probably already been detected in surveys like FIRST and NVSS, and even shallow SKA surveys will trivially find them all. However, [6] have shown that high signal-to-noise ratio spectroscopic observations are needed to measure reliable redshifts for these objects (from the forest of $\mathrm{HI} 21 \mathrm{~cm}$ absorption lines - the radio analogue of the Gunn-Peterson effect) and since they will be rare, and very hard to find in other ways, they will perhaps be both found and studied using an 'all-hemisphere' SKA survey (figure 5).

Carilli et al. 6] have argued that studies of the IGM within the EoR are made possible by HI $21 \mathrm{~cm}$ absorption against distant radio-loud sources. Such objects are also important in themselves as they probably contribute significantly to the heating of the IGM, its magnetization and the distribution of heavy elements during the EoR. Their influence on the ionized component of the cosmic web is likely to be profound, e.g. [16], and their jets carry enough kinetic power that they dominate the mechanical output of all accreting black holes. A single radio-loud outburst can, for example, shut-down star-formation and black-hole activity in $\sim 10-100$ surrounding protogalaxies in a protocluster 35.

Radio-quiet quasars are the dominant source of photoionizing (if not kinetic) power associated with accreting SMBH. They always have some radio emission, e.g. from star formation and weak jets. The SKA has sufficient sensitivity to detect essentially all this population within the EoR (Figure 6). Although finding these objects represents a challenging sifting problem, large FOV (c.f. present and future X-ray and mid-IR satel-

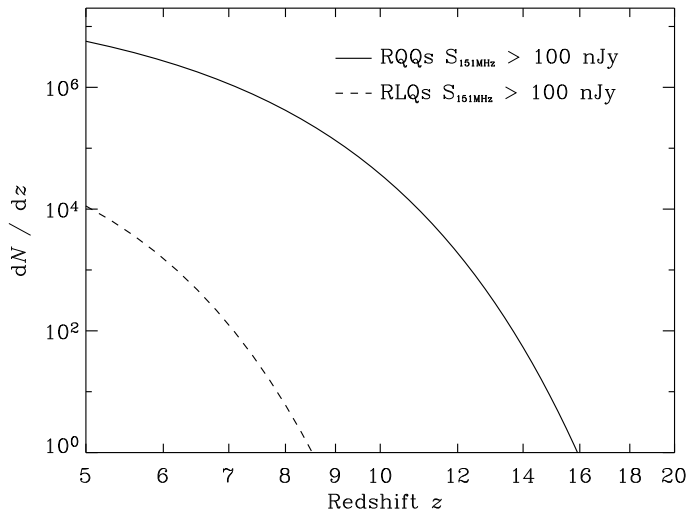

Figure 5. The predicted differential number $\mathrm{dN} / \mathrm{dz}$ of 'radio-loud' quasars (dashed line) and 'radio-quiet' QSOs (solid line) in an allhemisphere $(2 \pi)$, 1-year SKA survey at $\approx 0.15$ $\mathrm{GHz}$ (assuming a $5 \sigma$ continuum detection limit of $\approx 100 \mathrm{nJy}$, a $F O V=1 \mathrm{deg}^{2}$ at $\nu=1.4 \mathrm{GHz}$, scaling as $\nu^{-2}$ ). The prediction follows from the methods outlined in Jarvis \& Rawlings (this volume) and are consistent with the cosmic evolution in AGN trigger rates inferred from Fig. 6. All Radio-loud quasars [defined here as all objects, including optically-obscured 'radio galaxies'], in the sub-population dominating the radio luminosity function (RLF) at and above its characteristic break 44] will be detected at $>$ mJy levels and will therefore have high signal-to-noise SKA spectra, allowing studies of the IGM during the EoR using $\mathrm{HI}$ absorption 6. The SKA sensitivity is also sufficient to detect all the radio-quiet quasars in the EoR [defined here as all quasars, including optically-obscured 'Type-II' objects], at or above the characteristic break in the $\mathrm{X}$-ray luminosity function 40]. 


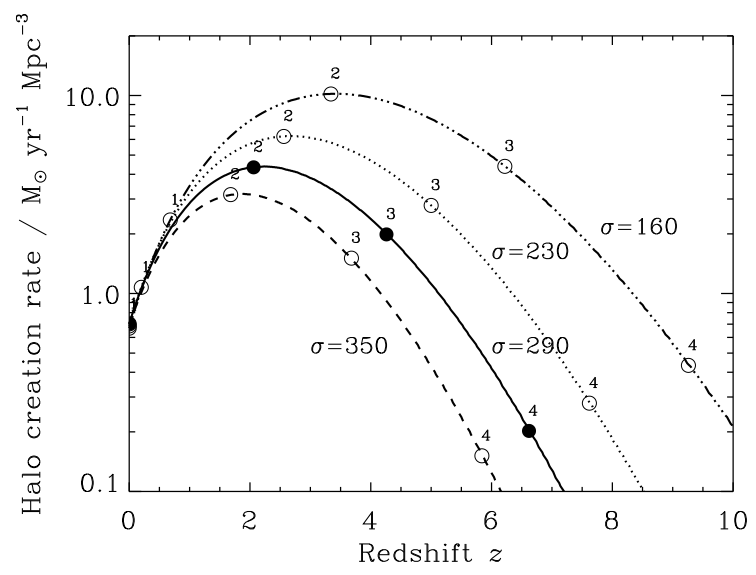

Figure 6. The creation rate of dark-matter halos as a function of redshift $z$ for various halo velocity dispersions $\sigma$ (in units of $\mathrm{km} \mathrm{s}^{-1}$ ) from Rawlings \& Jarvis [35]. Adopting the feedback model (for powerful jets) of Rawlings \& Jarvis, the halos capable of hosting these 'radio-loud' objects lie in the range $230 \leq \sigma \leq 350$, which correspond to $\nu=4$ (where $\nu$, marked by circles, is the density threshold for collapse in units of the r.m.s. density fluctuations) at $z \sim 6-8$. Adopting the feedback model (for weak jets) of Silk \& Rees (1998), the halos capable of hosting these 'radio-quiet' objects reach down to $\sigma=160$, and hence can form from a $\nu=4$ fluctuation at $z \sim 9.5$. lites) and (via deep SKA exposures) redshift measurement using associated HI $21 \mathrm{~cm}$ (rather than metal) lines, may prove crucial in finding the first (i.e. highest-redshift) SMBHs in the Universe.

\subsection{Compact radio sources}

In order to find the first SMBHs, besides its sensitivity and powerful surveying capability, the SKA can make additional use of its resolving power for identifying AGN. Almost every AGN, be it radio-loud or radio-quiet, is associated with a high brightness temperature radio core (this is discussed in more detail by Falcke, Körding, \& Nagar in this volume). The radio core is typically the inner part of a moderately relativistic plasma jet. At flux density levels of several mJy these radio cores are almost exclusively found to be associated with the SMBH in a galaxy. They stand out when using a spatial filter concentrating on the longest baselines of the telescope array.

Radio cores have been used effectively to identify AGN in wide field VLBI images of deep fields and for other AGN surveys. They are even more pronounced for low-power sources. Surveying the Palomar sample of nearby galaxies at a shallow level with the VLA, Nagar et al. 3031 showed that $\sim 40 \%$ of nearby AGN host a compact radio core that is easily detected and which reliably identifies AGN activity. At lower resolution, including extended emission, the detection rate even approaches $85 \%$ [19].

Compact radio core emission seems to be a function of accretion power and is related to the X-ray emission and the black hole mass. Black holes - stellar and supermassive - form a loosely defined fundamental plane in the parameter space opened up by radio flux, X-ray flux, and black hole mass 1027 . This relation implies that supermassive black holes are most effectively detected in the radio while $\mathrm{X}$-rays are more effective for stellar mass black holes.

Figure 7 shows the local radio core luminosity function of AGN (most of which are traditionally classified as radio-quiet) from Seyferts galaxies, via Low-Luminosity AGN down to our own Milky Way. The scale on the top shows the typical redshift range detectable for such AGN with the SKA in one hour. It shows that the SKA will allow the 
study of a very wide range of AGN activity with low-luminosity AGN being easily visibly out to $\mathrm{z}=1$ and more luminous ones even further. Besides studying AGN evolution, the SKA will thus also penetrate deep into the EoR. Even without beaming, radio cores are expected to be visible at $z>10$ from $10^{7} M_{\odot}$ black holes shining at their Eddington limit or from $10^{9} M_{\odot}$ black holes shining at $0.1 \%$ of the Eddington limit. This allows one to detect the very first SMBHs already in the process of their formation - if this happens through baryonic accretion.

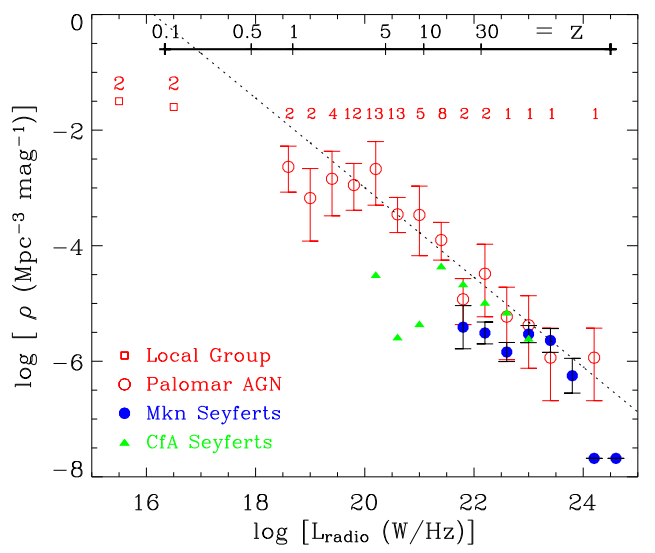

Figure 7. The $15 \mathrm{GHz}$ nuclear radio luminosity function (RLF) of the 150 mas-scale radio nuclei in the Palomar sample (red open circles, with the number of galaxies in each bin listed above the symbol) as compared to the RLFs for Markarian Seyferts and CfA Seyferts. The dashed line is a power-law $(-0.78)$ fit to the Palomar RLF. Also shown is the estimated nuclear RLF of galaxies in the local group. The upper scale illustrates the detection limits of the SKA (using an r.m.s noise of $0.3 \mu \mathrm{Jy}$ in $1 \mathrm{hr}$ of imaging, and a $3 \sigma$ detection threshold) of similar AGNs at redshifts of $z=$ 0.1-30 (Figure from [32], see also Falcke, Körding, Nagar, this volume).

The compact radio emission may, however, be further amplified if one does not only consider the inner, fairly dissipationless part of the jet. One could argue that the terminus of jets, i.e., their hot spots, from the first black holes will be confined to the host galaxy due to large ambient densities and pressures in the violent formation process of the early galaxies. Since the hot spots are working surfaces of the jet, their emission can dominate the overall radio emission easily and the compactness of the emission makes them appear as high-brightness temperatures sources.

The radio cores will then have a very characteristic, peaked broad band spectrum (called Gigahertz-Peaked Spectrum/GPS sources [33]) caused by synchrotron absorption with a doublelobed spatial structure on a scale of some tens of milli-arcsecond. This characteristic peak allows for designing very effective large survey capabilities as the physical size and the peak frequency are related: the smaller the source the higher the frequency. Fortuitously frequency and angular size show a very different behaviour as one goes to large redshifts: frequency will decrease while angular sizes become larger again for a standard ruler at $z>1$. Hence in the parameter space of angular size, peak frequency, and flux density, ultra-high redshift GPS sources should occupy a unique region (lower left corner in Fig. 8). They will have peak frequencies in the $0.1-0.3 \mathrm{GHz}$ regime, sizes of some 10-100 milli-arcsecond, and flux densities in the mJy regime.

An all-sky broad-band survey with highresolution follow-up observations should therefore very effectively produce candidates for the first SMBHs in the universe. Some of them may even be bright enough to do HI absorption spectroscopy in deep integrations to confirm their high-z nature. The crucial and qualitatively new features of the SKA to make this succeed are its huge instantaneous band-width to measure spectral curvature and its multi-beaming capability to facilitate large surveys.

In summary, the SKA seems to be an ideal instrument to study AGN evolution at all luminosity levels and all redshifts largely unaffected by dust obscuration. It even provides a number of angles on how to identify and study in detail the very first generation of SMBHs. As such the SKA 


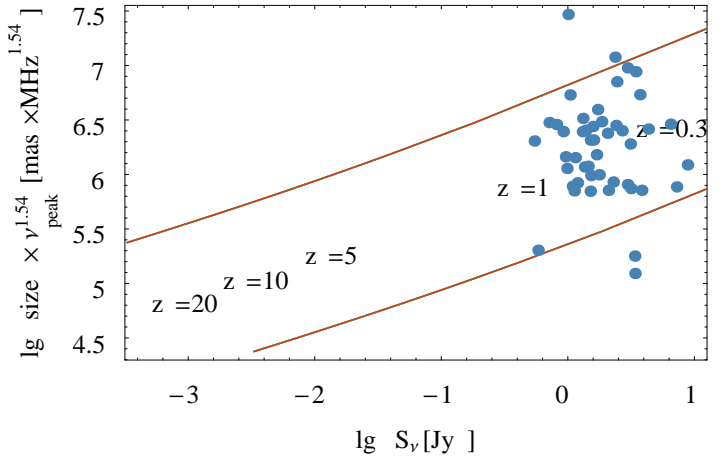

Figure 8. Size, frequency, and flux density roughly form a fundamental plane for GPS radio galaxies. Here we collapse this plane by plotting a combination of frequency and size $\left(\nu_{\text {peak }}^{1.54} \times r\right)$ vs. the flux density for a range of empirical GPS models and actual GPS sources. The two lines delimit the scatter in the empirical relation. The labels indicate the typical redshift of sources in that regime. Standard GPS sources are found in the redshift range around $z=1$. The bottom left corner is not occupied and is the discovery space for young high-redshift black holes with the SKA (Figure from Falcke, Körding, Nagar, this volume). will be an invaluable tool for cosmology and black hole physics alike.

\section{REFERENCES}

1. Bertoldi, F., Cox, P., Neri, R. et al. 2003, A\&A, 409, L47

2. Bertoldi, F., Carilli, C., Cox, P. et al. 2003, A\&A, 406, L15

3. Carilli, C. \& Blain, A. 2002, ApJ, 569, 605

4. Carilli, C., Walter, F., Bertoldi, F. et al. 2004a, AJ, submitted

5. Carilli, C., Bertoldi, F., Walter, F. et al. 2004b, in Multiwavelength AGN Surveys, eds. Maiolino and Mujica (World Scientific), in press (astroph/0402573)

6. Carilli, C., Gnedin, N., Owen, F. 2002, ApJ, 577,22

7. Cen, R. 2003, ApJ, 591, 12

8. Collin, S., Boisson, C., Mouchet, M., Dumont, A.-M., Coupe, S., Porquet, D., Rokaki, E., 2002, A\&A, 388, 771

9. Falcke, H., Nagar, N. M., Wilson, A. S., \& Ulvestad, J. S. 2000, ApJ, 542, 197

10. Falcke, H., Körding, E., \& Markoff, S. 2004, A\& A., 414, 895

11. Fan, X., Strauss, M., Schneider, D. et al.2003, AJ, 125, 1649

12. Furlanetto, S. \& Loeb, A. 2003, ApJ, 588, 18

13. Furlanetto, S., Zaldarriaga, M., Hernquist, L. 2004, ApJ, submitted (astroph/0403697)

14. Gnedin, N. 2004, ApJ, in press (astroph/0403699)

15. Gnedin, N. \& Shaver, P. 2003, ApJ, in press (astroph/03012005)

16. Gopal-Krishna, Wiita, P. J., 2001, ApJ, 560L, 115

17. Haiman, Z., Quatert, E., Bower, G. 2004, ApJ, in press (astroph/0403104)

18. Harford, A., \& Gnedin, N. 2003, ApJ, 597, 74

19. Ho, L. C. \& Ulvestad, J. S. 2001, ApJ (Supp), 133,77

20. Hopkins, A., Windhorst, R., Cram, L., Ekers, R. 2000, Experimental Astronomy, 10, 419

21. Hu, E., Cowie, L., McMahon, R. et al. 2002, ApJ, 568, L75

22. Hui, L. \& Haiman, Z. 2003, ApJ, 596, 9

23. Iliev, I., Scannapieco, E., Martel, H., Shapiro, 
P. 2003, MNRAS, 341, 81

24. Iliev, I., Shapiro, P., Ferrara, A., Martel, H. 2002, ApJ, 572, L123

25. Kodaira, K., TAniguchi, Y., Kashikawa, N. 2003, PASJ, 55, L17

26. Kogut, A., Spergel, D., Barnes, C. et al. 2003, ApJS, 148, 161

27. Merloni, A., Heinz, S., \& di Matteo, T. 2003, MNRAS, 345, 1057

28. Mesinger, A. \& Haiman, Z. 2004, ApJ (letters), in press (astroph/0406188)

29. Morales, M. \& Hewitt, J. 2004, ApJ, in press (astroph/0312437)

30. Nagar, N. M., Falcke, H., Wilson, A. S., \& Ho, L. C. 2000, ApJ, 542, 186

31. Nagar, N. M., Falcke, H., Wilson, A. S., \& Ulvestad, J. S. 2002, A\& A, 392, 53

32. Nagar, N. M., et al, 2004, submitted to A\&A

33. O'Dea C. P., 1998, ASP, 110, 493

34. Pello, R., Schaerer, D., Richard, J. et al. 2004, A\&A, 416, L35

35. Rawlings, S. \& Jarvis, M.J., 2004, MNRAS, in press

36. Richards, G.T., vanden Berk, D., Reichard, T. 2002, AJ, 124, 1

37. Shaver, P., Windhorst, R., Madau, P., de Bruyn, G. 1999, A\& A, 345, 380

38. Silk, J. \& Rees, M.J., 1998, A\&A, 331, L1

39. Stanway, E., Glazebrook, K., Bunker, A. et al. 2004, ApJ 604, L13

40. Ueda et al., 2003, ApJ, 598, 886

41. Walter, F., Bertoldi, F., Carilli, C. et al. 2003, Nature, 424, 406

42. Weatherley, S., Warren, S., Babbedge, T. 2004, MNRAS, submitted (astroph 0407150)

43. White, R., Becker, R., Fan, X., Strauss, M. 2003, AJ, 126, 1

44. Willott, C.J., Rawlings, S., Blundell K.M., Lacy M., \& Eales, S.A., 2001, MNRAS, 322, 536

45. Willott, C.J., McClure, R., \& Jarvis, M. 2003, ApJ, 587, L15

46. Withe, A. \& Loeb, L. 2004, Nature, 427, 815

47. Zaldarriaga, M., Furlanetto, S., Hernquist, L. 2004, ApJ, in press (astroph/0311514) 


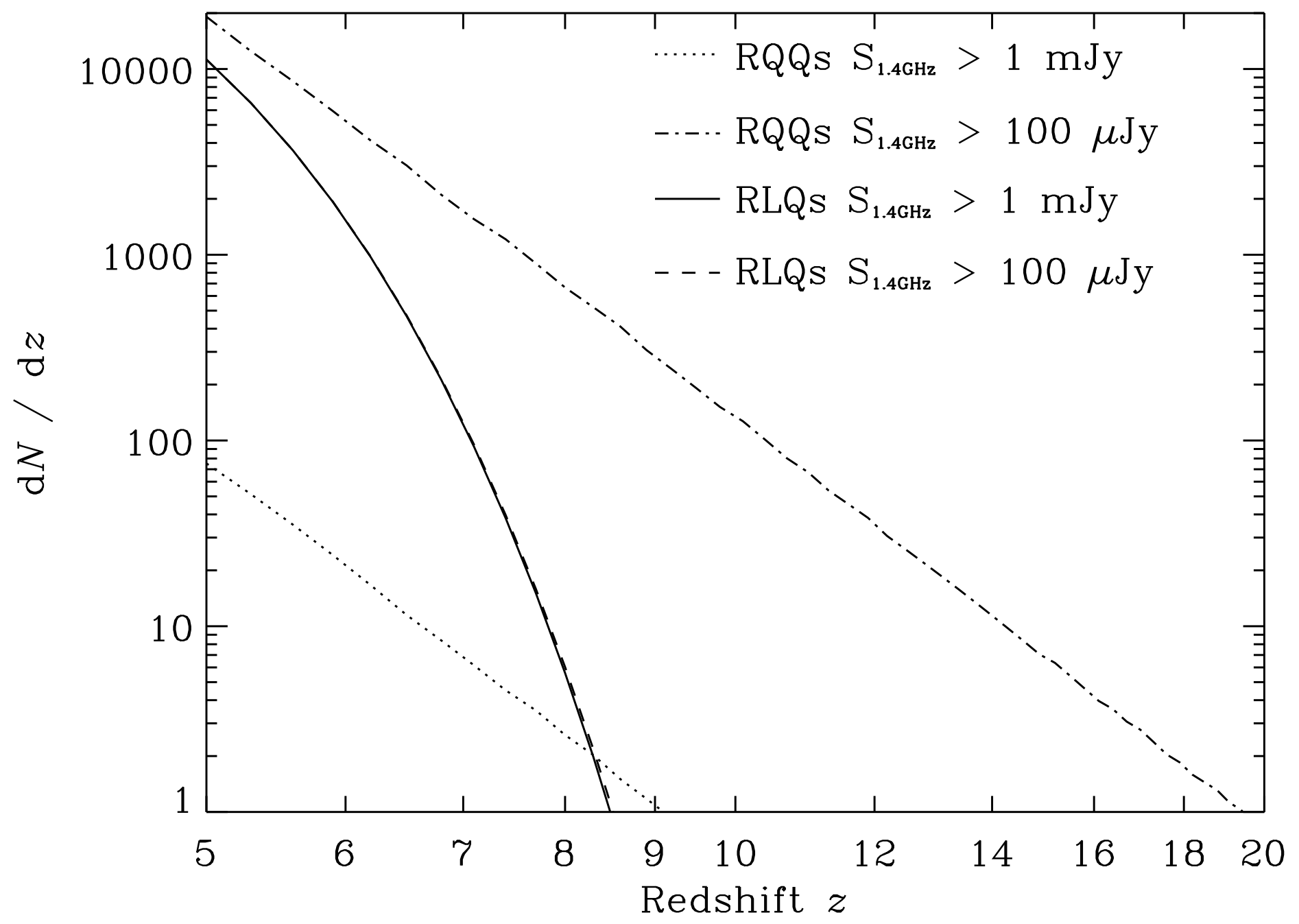




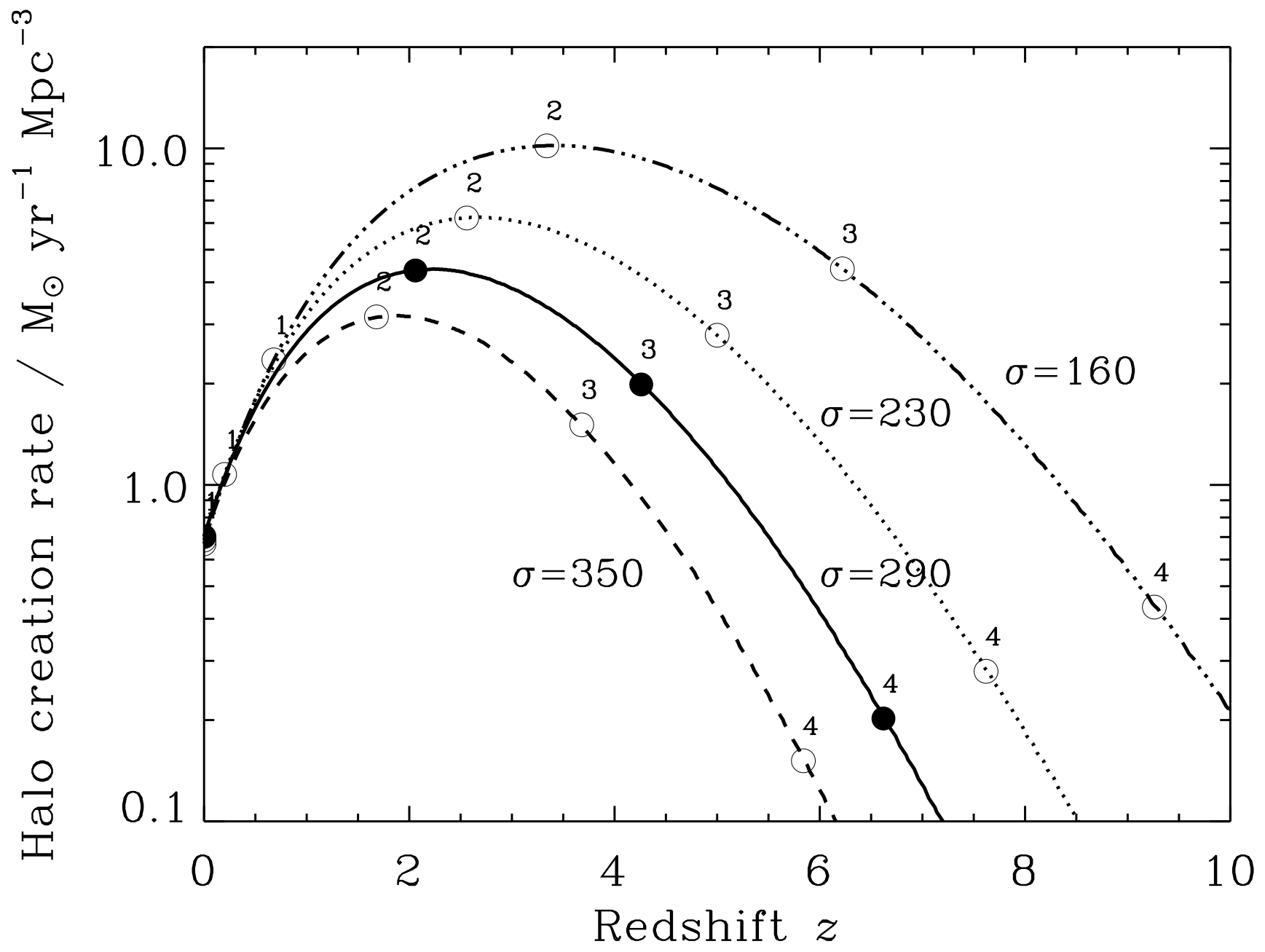




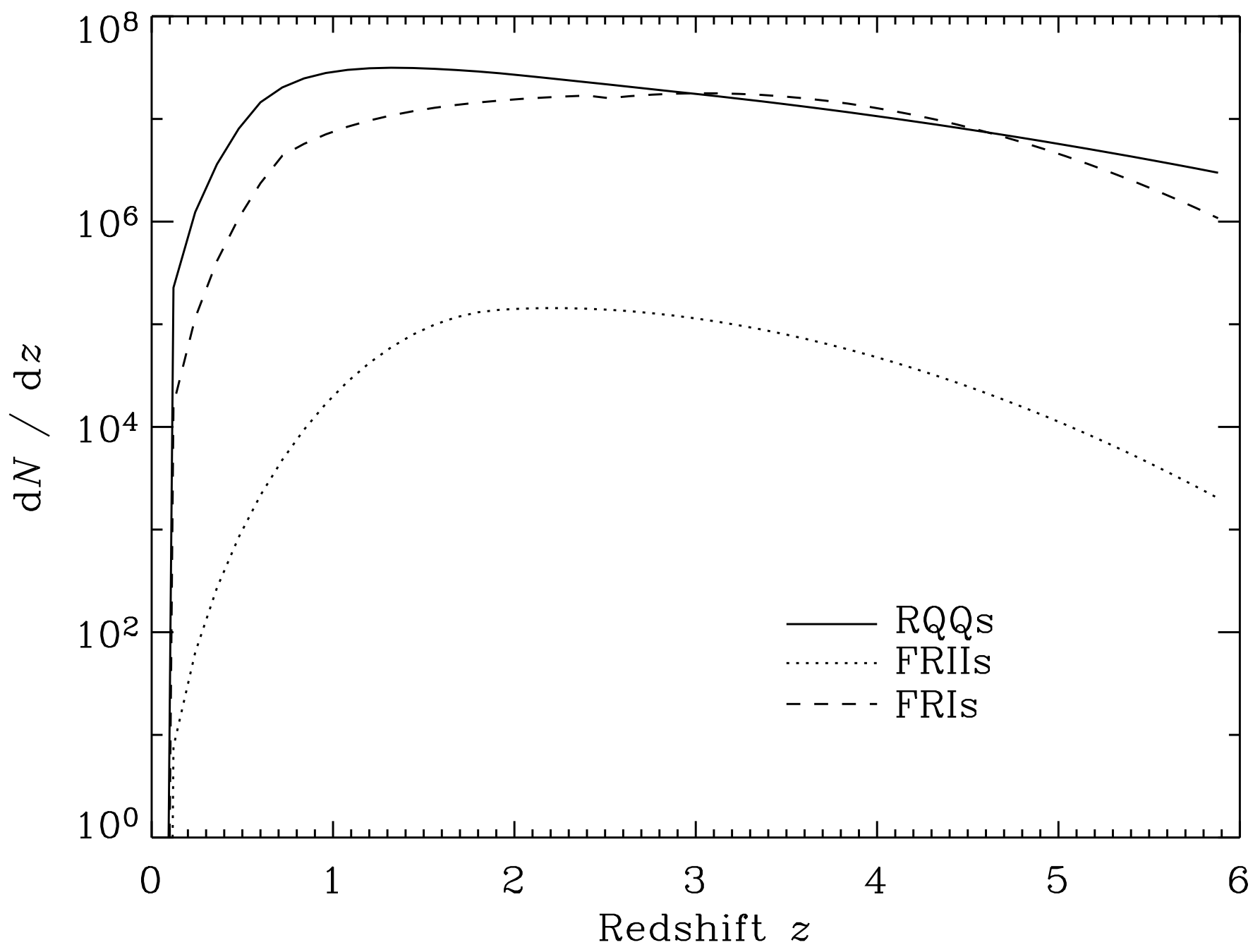


This figure "Arp220.SKA.jpg" is available in "jpg" format from: http://arxiv.org/ps/astro-ph/0409312v2 
This figure "rlfpalvsmkn.jpg" is available in "jpg" format from: http://arxiv.org/ps/astro-ph/0409312v2 